Introduction The definition of pesticide covers about 100000 different chemicals, inorganic and organic, that are grouped according to their function or their chemical structure. Evidence is growing about long-term health effects following high-level, long-lasting exposure to some pesticides, including asthma and other allergic diseases, immunotoxicity, endocrine disruption, cancer, and central and peripheral nervous system effects. Identifying such specific chemicals is of paramount importance in preventing ill health in farmers, while preserving the benefits of plant protection against pests.

Methods An extensive review of the literature was conducted. Result Currently, the International Agency for Research on Cancer classifies three obsolete agrochemicals as Group 1 human carcinogens (Arsenic compounds, lindane, and 1,2dichloropropane); six are classified as probable human carcinogens, including captafol, DDT, diazinon, glyphosate, ethylene dibromide, and malathion, generating a vast controversy among the International Health and Safety Agencies and the media about the need to phase them out as some were widely used worldwide. Possibly human carcinogen pesticides are 20, and for 32 there is inadequate scientific evidence to classify their potential human carcinogenicity. The endocrine disrupting effects of numerous pesticides have also been extensively studied, but evidence of hormonal effects comes mainly from animal studies, either laboratory or wildlife animals; such results are difficult to refer to the low level long term exposure in humans. Other chronic effects repeatedly reported among users of specific pesticides include asthma and chronic bronchitis, immunosuppression, and neurodegenerative diseases, with the classical example of paraquat, but also other pesticide classes, and Parkinson's disease.

Discussion Major reasons for uncertainty in interpreting epidemiological findings of long term pesticide effects include the complex pattern of overlapping exposure due to multiple treatments applied to different crops and their frequent changes over time to overcome pest resistance. Further research will have to address specific agrochemicals with wellcharacterised exposure patterns.

\section{9d INDISCRIMINATE USE OF PESTICIDES IN AGRICULTURE AND EFFECTS ON HEALTH AND ENVIRONMENT EXAMPLES FROM BOLIVIA, UGANDA, NEPAL AND CAMBODIA!}

E Jørs. Department of Occupational and Environmental Health, Odense University Hospital, Denmark

\subsection{6/oemed-2018-ICOHabstracts. 1333}

Introduction Pesticide use is increasing in developing countries trying to boost their agricultural production. Due to lack of proper knowledge among small-holder farmers this leads to frequent poisonings of farmers and pollution of the environment. Integrated Pest Management (IPM) has been tried out to minimise the use of pesticides and a banning of the most toxic pesticides is seen in some countries.

Methods Small-holder farmers from Bolivia, Nepal, Uganda and Cambodia were interviewed in cross-sectional baseline studies before an intervention teaching farmers IPM took place. Follow-up studies were conducted after training was completed to evaluate the effect of the trainings. Sound statistical methods were applied while controlling for relevant confounders. Ethical approval was obtained.
Results At baseline farmers used generally very toxic pesticides, most pronounced in Bolivia. Farmers had little knowledge and use of personal protection when handling pesticides, and use of alternatives to pesticides were scarce. Pesticide poisonings were commonly reported and an effect with a lowered blood level of AChE was seen depending on spraying frequency, use of PPE and the toxicity of the pesticides used. Training improved farmer's knowledge and practice on pesticide handling and IPM and lowered the number of symptoms of poisoning after spraying. Obstacles for a wider diffusion of IPM seem to be an extra workload not always compensated for by higher prices of the products.

Conclusion Pesticide poisonings is increasing among farmers from low income countries, leading to acute symptoms eventually dead or chronic poisonings with neurological impairment, cancer, lung diseases or skin diseases and fetal damage. Pesticide abuse can be prevented without the loos of crops by teaching farmers integrated pest management methods and political interventions with a ban of the most dangerous pesticides nationally and worldwide have proved effective as well.

\section{9e PESTICIDE PREVENTION STARTS FROM THE PRE- MARKETING PHASE}

A Moretto. Department of Biomedical and Clinical Sciences, University of Milan, Italy

\subsection{6/oemed-2018-ICOHabstracts.1334}

Pesticide active ingredients are extensively studied before being authorised for use. These include, among others, efficacy studies, several toxicity studies in mammals and in ecotoxicological relevant species. From these studies, health based limits for the consumer are derived such as the acceptable daily intake (ADI), the acute reference dose (ARfD) and the exposure limits for pesticide operators (AOEL); also, environmental limits are defined and levels of residues in food commodities determined.

Then the exposure for consumer and operators is estimated by using different mathematical models based on the proposed uses scenario (e.g: application doses, period and interval between application, etc). A further step is to compare the estimated exposure with the reference value to assess the risk and conclude if the proposed use is safe, therefore the active ingredient can be put on the market, or not. It has to be underlined that the use scenario for exposure estimation is always based on a worst-case approach to cover all possible conditions.

In particular, estimation of operators' exposure is made on mathematical models based on dermal and inhalation exposures measured following different type of application in several field studies.

Finally, if safe uses have been identified, approval of an active ingredient and of a commercial formulation is consequently accompanied by indication of the proper application modalities and PPE to be used by the pesticide applicator to reflect the scenario evaluated.

Moreover, the risk assessment is conducted not only for consumer and operators but also for workers re-entering the treated crops, bystander, and especially resident.

Therefore, it can be concluded that if an active ingredient is used on crops according the Good Agricultural Practice and according all indications on the label (e.g.: use of specific PPE, buffer zone, post harvesting interval), negligible risk for human and environment is expected. 